\title{
On the status of the hoop conjecture in charged curved spacetimes
}

\author{
Shahar Hod ${ }^{1,2, a}$ \\ ${ }^{1}$ The Ruppin Academic Center, 40250 Emeq Hefer, Israel \\ 2 The Hadassah Academic College, 91010 Jerusalem, Israel
}

Received: 8 August 2018 / Accepted: 4 December 2018 / Published online: 13 December 2018

(c) The Author(s) 2018

\begin{abstract}
The status and regime of validity of the famous Thorne hoop conjecture in spatially regular charged curved spacetimes are clarified.
\end{abstract}

\section{Introduction}

The hoop conjecture [1] has attracted the attention of physicists and mathematicians since its introduction by Thorne almost five decades ago [1,2]. This mathematicallys elegant and physically influential conjecture asserts that a selfgravitating matter configuration of mass $M$ will form an engulfing horizon if its circumference radius $R=C / 2 \pi$ is equal to (or less than) the corresponding Schwarzschild radius $2 M$ [3]. That is, the hoop conjecture states that [1]

$C \leq 4 \pi M \Longrightarrow$ Black-hole horizon exists .

It is widely believed that the hoop conjecture reflects a fundamental aspect of classical general relativity. In particular, the conjecture is supported by several studies (see [46] and references therein). Intriguingly, however, there are also some claims in the physics literature that the Thorne hoop conjecture can be violated in charged curved spacetimes $[7,8]$.

The main goal of the present compact paper is to clarify the status of the Thorne hoop conjecture in spatially regular charged spacetimes. In particular, below we shall explicitly demonstrate that the hoop conjecture is valid in charged curved spacetimes provided the mass parameter on the r.h.s of the hoop relation (1) is appropriately interpreted as the gravitational mass $M(R)$ contained within the engulfing hoop of radius $R$ and not as the total (asymptotically measured) mass $M_{\infty}$ of the entire spacetime.

a e-mail: shaharhod@gmail.com

\section{Validity of the hoop conjecture in spatially regular charged spacetimes}

It has been argued in $[7,8]$ that the hoop conjecture (1) can be violated in spatially regular horizonless charged spacetimes. In particular, Ref. [7] analyzed the compactness of spherically symmetric fluid matter configurations with uniform charge densities and concluded that, taking the mass parameter in the r.h.s of the hoop relation (1) as the total mass $M_{\infty}$ of the system (as measured by asymptotic observers), the hoop conjecture can be violated. As an illustrative example, Ref. [7] constructed a uniformly charged horizonless ball which is characterized by the dimensionless relations

$\frac{M_{\infty}}{R}=0.65$ and $\quad \frac{Q^{2}}{R^{2}}=0.39$

This spatially regular horizonless charged matter configurations is characterized by the dimensionless ratio

$\frac{C}{4 \pi M_{\infty}} \simeq 0.769<1$

and, as claimed in [7], it therefore violates the hoop conjecture (1).

However, we believe that in the Thorne hoop conjecture (1), which relates the mass parameter of the system to its circumference radius $R=C / 2 \pi$, it is physically more appropriate to interpreted $M$ as the gravitational mass contained within the engulfing hoop and not as the total mass of the entire curved spacetime.

In particular, since the exterior $(r>R)$ electromagnetic energy density associated with a charged ball of radius $R$ and electric charge $Q$ is $T_{0}^{0}(r>R)=Q^{2} / 8 \pi r^{4}$ [9], the electromagnetic energy $E_{\text {elec }}(r>R)=\int_{R}^{\infty} T_{0}^{0} 4 \pi r^{2} d r$ outside the charged ball is given by the simple expression

$E_{\mathrm{elec}}(r>R)=\frac{Q^{2}}{2 R}$. 
Thus, for a charged ball of radius $R$, electric charge $Q$, and total mass (energy) $M_{\infty}$ as measured by asymptotic observers, the gravitational mass contained within $(r \leq R)$ the ball is given by

$M(r \leq R)=M_{\infty}-\frac{Q^{2}}{2 R}$

From Eqs. (2) and (5) one obtains the dimensionless relation

$\frac{M(r \leq R)}{R}=0.455$

for the horizonless charged matter configuration considered in [7]. Taking cognizance of Eqs. (1) and (6), one finds

$\frac{C(R)}{4 \pi M(r \leq R)} \simeq 1.099>1$.

The dimensionless ratio (7) implies, in particular, that the uniformly charged matter configurations studied in [7] do not violate the Thorne hoop conjecture (1).

\section{Summary}

In this compact paper we have explored the (in)validity of the Thorne hoop conjecture [1] in spatially regular charged curved spacetimes. Our analysis is motivated by the intriguing claims made in the physics literature (see e.g. [7,8]) according to which this famous conjecture, which is widely believed to reflect a fundamental aspect of classical general relativity, can be violated by horizonless charged matter configurations.
The present analysis clearly demonstrates the fact that, as opposed to the claims made in $[7,8]$, the Thorne hoop conjecture is valid in charged spacetimes provided that, for a given radius $R$ of the engulfing hoop, the mass parameter in the hoop relation (1) is appropriately interpreted as the gravitational mass $M(R)$ contained within the hoop (sphere) of radius $R$ and not as the total mass $M_{\infty}$ of the entire spacetime.

Acknowledgements This research is supported by the Carmel Science Foundation. I would like to thank Yael Oren, Arbel M. Ongo, Ayelet B. Lata, and Alona B. Tea for stimulating discussions.

Open Access This article is distributed under the terms of the Creative Commons Attribution 4.0 International License (http://creativecomm ons.org/licenses/by/4.0/), which permits unrestricted use, distribution, and reproduction in any medium, provided you give appropriate credit to the original author(s) and the source, provide a link to the Creative Commons license, and indicate if changes were made. Funded by SCOAP $^{3}$.

\section{References}

1. K.S. Thorne, in Magic Without Magic: John Archibald Wheeler, ed. by J. Klauder (Freeman, San Francisco, 1972)

2. C.W. Misner, K.S. Thorne, J.A. Wheeler, Gravitation (Freeman, San Francisco, 1973)

3. We shall use natural units in which $G=c=\hbar=1$

4. I.H. Redmount, Phys. Rev. D 27, 699 (1983)

5. A.M. Abrahams, K.R. Heiderich, S.L. Shapiro, S.A. Teukolsky, Phys. Rev. D 46, 2452 (1992)

6. S. Hod, Phys. Lett. B 751, 241 (2015). arXiv:1511.03665

7. J.P. de León, Gen. Relativ. Grav. 19, 289 (1987)

8. W.B. Bonnor, Phys. Lett. A 99, 424 (1983)

9. K. Gottfried, V.F. Weisskopf, Concepts of Particle Physics, vol. 2 (Oxford University Press, Oxford, 1986) 